\title{
A retrospective analysis of plasma concentration monitoring of fluorouracil in patients with advanced colorectal cancer
}

\author{
Quanliang Yang, ${ }^{1}$ Yanzhi Bi, ${ }^{1}$ Xiaogian $\mathrm{Li}^{1}$ Qian Liu, ${ }^{2}$ Jian Ma, ${ }^{1}$ Chengliang Zhang, ${ }^{3}$ \\ Jinlin Zhang, ${ }^{4}$ Guangzhao He ${ }^{5}{ }^{5}$
}

'Oncology, Changzhou Cancer Hospital, Soochow University, Changzhou, China

Laboratory Medicine, Changzhou Cancer Hospital, Soochow University, Changzhou, China ${ }^{3}$ Pharmacy, Tongji Hospital of Tongji Medical College of Huazhong University of Science and Technology, Wuhan, China ${ }^{4}$ Pharmacy, Affiliated Cancer Hospital of Nantong University, Nantong, China

${ }^{5}$ harmacy, Changzhou Cancer Hospital, Soochow University, Changzhou, China

\section{Correspondence to}

Dr Guangzhao He, Pharmacy,

Changzhou Cancer Hospital,

Soochow University,

Changzhou 213032, China;

shmily_hgzhao@hotmail.com

QY and YB contributed equally.

QY and $Y B$ are joint first authors.

Received 10 January 2019 Revised 15 February 2019 Accepted 19 February 2019

Published Online First

16 March 2019

EAHP Statement 4: Clinical Pharmacy Services.

\section{ABSTRACT}

Objectives To analyse the results of fluorouracil (5-

FU) plasma concentration monitoring in patients with advanced colorectal cancer after 5-FU treatment, and to provide a reference for the application prospect of 5-FU plasma concentration monitoring technology.

Methods A retrospective analysis was performed with advanced colorectal cancer patients treated with 5-FU from March 2015 to August 2018. The results of plasma concentration monitoring of 5-FU, severe adverse reactions, and anti-tumour efficacy were analysed.

Results Among 47 patients, 5-FU plasma concentration monitoring was carried out a total of 289 times. The area under the receiver operating characteristic (ROC) curve (AUC) reflecting 5-FU exposure in vivo was $2.8-158 \mathrm{mg}{ }^{*} \mathrm{~h} / \mathrm{L}\left(41 \pm 94.6 \mathrm{mg}{ }^{*} \mathrm{~h} / \mathrm{L}\right)$. Mean AUC range within the target range $\left(20-30 \mathrm{mg}{ }^{*} \mathrm{~h} / \mathrm{L}\right)$ for each patient was observed in $28.8 \%$ of patients. The overall incidence of related severe adverse reactions in the AUC $\leq 30 \mathrm{mg}{ }^{*} \mathrm{~h} / \mathrm{L}$ group was lower than that in the $>30 \mathrm{mg}{ }^{*} \mathrm{~h} / \mathrm{L}$ group $(24.0 \%$ and $50.0 \%$, respectively) $(p=0.06)$, and the incidence of severe neutropenia was $12.0 \%$ and $40.9 \%$, respectively $(p=0.05)$. The disease control rate and overall response rate of the AUC $<20$ $\mathrm{mg}{ }^{*} \mathrm{~h} / \mathrm{L}$ group was lower than that of the $\geq 20 \mathrm{mg}{ }^{*} \mathrm{~h} / \mathrm{L}$ group: $83.3 \%$ vs $97.1 \%(p=0.19)$ and $25.0 \%$ vs $51.4 \%$ $(p=0.10)$, respectively.

Conclusions The 5-FU plasma concentration monitoring technique can improve the safety and efficacy of 5-FU administration to advanced colorectal cancer patients. It is expected to become an important means to individualise 5-FU use in the Chinese population.

\section{INTRODUCTION}

Colorectal cancer is one of the top five malignant tumours in China; its high incidence and mortality are second only to lung, gastric, oesophageal and liver cancer. ${ }^{1}$ FOLFOX/FOLFIRI consists of fluorouracil (5-FU), oxaliplatin and irinotecan combined with bevacizumab, and is a first-line anti-tumour therapeutic regimen for advanced colorectal cancer. ${ }^{2}$ Due to the general condition of a patient, adverse drug reactions, patient medication compliance, and so on, the chemotherapy drug dose cannot be calculated solely based on body surface area or be empirically reduced in most cases. ${ }^{34}$ The evaluation of indexes such as dose index (DI), time index (TI) and relative dose intensity (RDI) can, however, objectively reflect patient acceptance of a chemotherapy drug dose. $^{35}$

\section{Key messages}

What is already known on this subject

- Monitoring fluorouracil (5-FU) plasma concentration has been used with some success in Europe, but much less so in China.

- Different dose adjustment regimens of 5-FU based on monitoring 5-FU plasma concentration is proposed by a number of non-Chinese publications.

What this study adds

- 5-FU plasma concentration monitoring may improve the safety and efficacy of treating patients with advanced colorectal cancer in China.

- Dose adjustment regimen of 5-FU based on monitoring 5-FU plasma concentration should be used in Chinese patients.

Monitoring 5-FU plasma concentration has been used with some success in Europe, and serves as an important technical means to individualise 5-FU drug dose. A series of studies by Gamelin et al found that when 5-FU area under the receiver operating characteristic (ROC) curve (AUC) is within the target range, namely $20-25$ or $20-30 \mathrm{mg} * \mathrm{~h} / \mathrm{L}$, an optimal anti-tumour effect with a relatively small toxic and side effect profile could be achieved. However, when 5-FU AUC is lower than the target, subsequent 5-FU dosage should be increased; otherwise, 5-FU dosage should be reduced. ${ }^{6-11}$

Since March 2015, our hospital has been using 5 -FU plasma concentration monitoring with the aim of exploring its reference value and comparing its clinical efficacy and safety for patients with advanced colorectal cancer in China. Herein, we reviewed all patients who underwent 5-FU plasma concentration monitoring from the initiation of this technology to August 2018. The 5-FU DI, plasma concentration monitoring results, severe adverse reactions and anti-tumour curative efficacy were retrospectively analysed to provide a reference for the potential application of the 5-FU plasma concentration monitoring technology in Chinese patients.

\section{DATA AND METHODS}

\section{Clinical data}

We selected patients with advanced colorectal cancer who received 5-FU therapy at our hospital 
Table 1 Results of fluorouracil plasma concentration monitoring

\begin{tabular}{lll}
\hline AUC level $\left(\mathbf{m g}^{*} \mathbf{h} / \mathbf{L}\right)$ & Case time $(\mathbf{n})$ & $\begin{array}{l}\text { Case } \\
\text { load }(\mathbf{n})\end{array}$ \\
\hline$<20$ & 117 & 12 \\
$20-30$ & 60 & 13 \\
$>30$ & 112 & 22 \\
Total & 289 & 47 \\
\hline
\end{tabular}

AUC, area under the receiver operating characteristic (ROC) curve.

from March 2015 to August 2018; basic data such as gender, age, body surface area, complicating diseases, chemotherapy regimen, and drug dosage were retrieved. The 5-FU was produced by Tianjin Jinyao Pharmaceutical Co., Ltd, State Food and Drug Administration approval number: H12020959 (specification: $10 \mathrm{~mL}, 0.25 \mathrm{~g}$ ).

Main inclusion criteria included patients: treated with mFOLFOX6, FOLFOR4 or FOLFIRI as the first-line or secondline therapeutic regimen; who received $\geq 2$ cycles of anti-tumour therapy and $\geq 15$-FU plasma concentration monitoring procedure during hospitalisation; and evaluated with radiographic tumour assessment at least once. Patients or their legal representative must have also given written informed consent. Main exclusion criteria included patients with incomplete medical records; who developed severe adverse reactions or complications, and refused further therapy; and patients with visibly poor treatment compliance.

\section{Drug dose}

Standard drug dose mFOLFOX6 and FOLFIRI regimen, the 5 -FU standard medication regimen, was $400 \mathrm{mg} / \mathrm{m}^{2}$, intravenous (iv), d1, 5-FU $2400 \mathrm{mg} / \mathrm{m}^{2}$, iv pump 46 hours. FOLFOX4 regimen was 5 -FU $400 \mathrm{mg} / \mathrm{m}^{2}$, iv, d1, d2, 5-FU $600 \mathrm{mg} / \mathrm{m}^{2}$ iv pump 22 hours, d1, d2. One treatment cycle spanned 14 days.

Dose evaluation index ${ }^{35}$

DI refers to the percentage of the actual drug dose in the standard drug dose. TI refers to the percentage of the standard

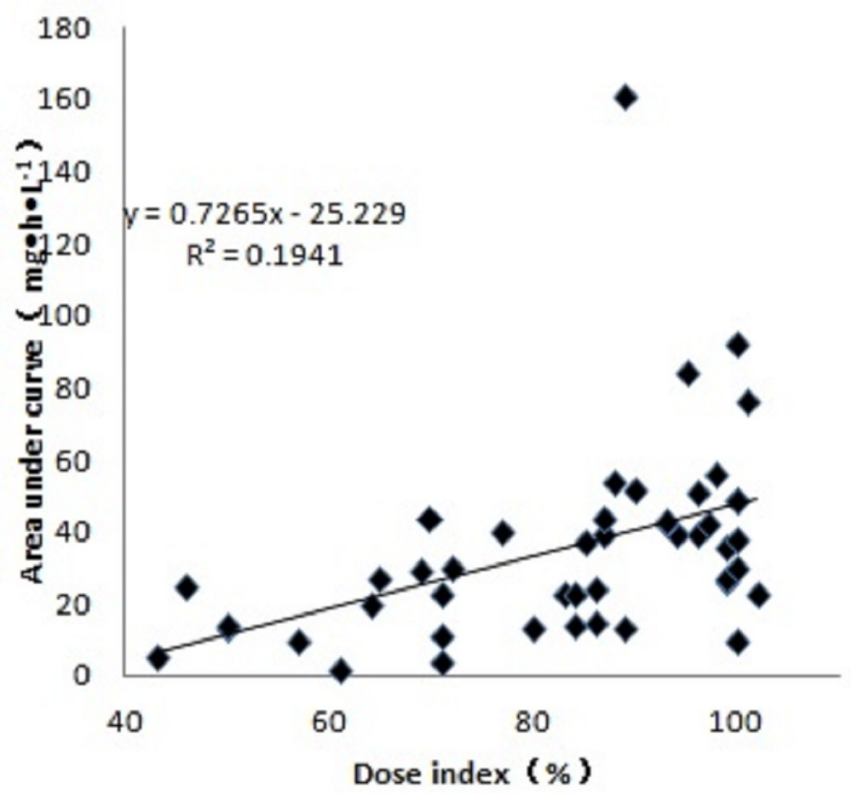

Figure 1 Linear analysis of fluorouracil dose index and area under the receiver operating characteristic (ROC) curve (AUC). drug use interval in the actual drug use interval. Dose intensity $=$ DI $\times$ TI. RDI refers to the percentage of the actual dose intensity of a specific drug in the standard dose intensity. For instance, for mFOLFOX6, as the standard dose intensity is 1400 $\mathrm{mg} / \mathrm{m}^{2} /$ week, and the actual dose intensity is $1200 \mathrm{mg} / \mathrm{m}^{2} /$ week, its RDI is $85.7 \%$.

\section{$\mathrm{DI}, \mathrm{TI}$ and RDI}

When determining initial DI, TI and RDI, factors such as general condition, age, body surface area, and willingness to accept treatment, and so on should be considered. Subsequent DI, TI and RDI should be based on 5-FU plasma concentration monitoring results, adverse reactions after medication and other factors; 5-FU drug dose was jointly decided by two experienced tumour physicians, and a clinical pharmacist assisted for some cases.

\section{5-FU plasma concentration monitoring \\ Plasma sample collection}

For the 5-FU pump, medication time was uniformly confirmed to start at 15:30-16:30 on day 1 . At 8:30-9:30 on day 3 from the 5-FU continuous intravenous pumping, venous blood of the patient was sampled and placed into a polyethylene tube pretreated with stabiliser (gimeracil) for $10 \mathrm{~min}$ by a nurse, then stored for 2 hours at $2^{\circ} \mathrm{C}-8^{\circ} \mathrm{C}$. Plasma was extracted by centrifugation, and cryopreserved at $-80^{\circ} \mathrm{C}$ for 3 months by a pharmacist.

\section{5-FU plasma concentration assay}

The 5-FU plasma concentration monitoring method established in the previous stage was used. ${ }^{12}$ In brief, patient venous blood was sampled and centrifuged to extract plasma, and plasma sample pretreated by liquid-liquid extraction. For chromatographic conditions, an Agela Innoval NH2 chromatographic column $(2.1 \mathrm{~mm} \times 50 \mathrm{~mm}, 5 \mu \mathrm{m})$ was used for isocratic elution in the fluid phase with methanol:ultrapure water $(2: 98)$ at a flow rate of $0.3 \mathrm{~mL} / \mathrm{min}$; column temperature was $40^{\circ} \mathrm{C}$. Using mass spectrometry, 5-FU and 5-bromouracil (the internal standard) were detected under a multi-reaction monitoring scan mode using electrospray negative ion mode. Quantitative analysis of ion pairs yielded $\mathrm{m} / \mathrm{z} 128.8 \rightarrow 42.1(5-\mathrm{FU})$ and $\mathrm{m} / \mathrm{z} 188.6 \rightarrow 42.1$ (5-bromouracil). The 5-FU plasma concentration was measured using a liquid chromatography tandem mass spectrometer (Shimadzu LC System Tandem AB Sciex API Model 3200 Triple Quadrupole Mass Spectrometer).

\section{5-FU plasma concentration monitoring results}

5-FU plasma concentration monitoring results were expressed by AUC. The calculation formula was as follows: AUC $\left(\mathrm{mg}^{*} \mathrm{~h} / \mathrm{L}\right)=5$ FU plasma concentration $(\mathrm{ng} / \mathrm{mL}) \times$ pump maintenance time (h)/1000. Based on reports by Kaldate and Denda for this subject group, we used AUC in the $20-30 \mathrm{mg}$ *h/L group as the target range. ${ }^{8}{ }^{10}$ AUC $<20 \mathrm{mg}$ *h/L indicated that anti-tumour therapy was not sufficiently effective; thus, increasing the subsequent 5 -FU dose may be necessary. AUC $>30 \mathrm{mg} * \mathrm{~h} / \mathrm{L}$ indicated an increased risk of severe adverse reaction; thus, reducing the subsequent 5-FU dose may be necessary. ${ }^{9}$ Mean AUC represents average AUC obtained per patient per time.

\section{Evaluation of adverse reactions}

The Common Terminology Criteria for Adverse Events (CTCAE) Version 4.03 standard was used as an evaluation criteria, and severe adverse events associated with 5-FU (including 
Table 2 The relationship between area under the curve (AUC) and treatment outcomes

\begin{tabular}{|c|c|c|c|c|c|c|c|c|c|c|c|}
\hline \multirow[b]{2}{*}{ AUC level } & \multicolumn{7}{|c|}{ Curative effect case load (n) } & \multicolumn{4}{|c|}{ Curative effect case load (n) } \\
\hline & Neutropenia & Anaemia & Thrombocytopenia & Diarrhoea & $\begin{array}{l}\text { Oral } \\
\text { mucositis }\end{array}$ & $\begin{array}{l}\text { Hand-foot } \\
\text { syndrome }\end{array}$ & $\begin{array}{l}\text { Cases having } \\
\text { severe adverse } \\
\text { reactions }(\mathrm{n})\end{array}$ & $\begin{array}{l}\text { Complete } \\
\text { response }\end{array}$ & $\begin{array}{l}\text { Partial } \\
\text { response }\end{array}$ & Stable & Progressive \\
\hline$<20$ & 1 & 0 & 0 & 0 & 0 & 0 & 1 & 0 & 3 & 7 & 2 \\
\hline $20-30$ & 2 & 0 & 1 & 1 & 1 & 0 & 5 & 0 & 5 & 7 & 1 \\
\hline$>30$ & 9 & 1 & 1 & 1 & 4 & 0 & 11 & 2 & 11 & 9 & 0 \\
\hline
\end{tabular}

AUC, area under the receiver operating characteristic (ROC) curve.

neutropenia, anaemia, thrombocytopenia, diarrhoea, oral mucositis, hand-foot syndrome) were statistically analysed. ${ }^{13}$

\section{Curative effect judgment criteria}

The curative effect was evaluated using the Response Evaluation Criteria in Solid Tumours (RECIST) Version 1.1, divided into complete response (CR), partial response (PR), stable disease $(\mathrm{SD})$ and progressive disease (PD). Disease control rate $(\mathrm{DCR})=(\mathrm{CR}+\mathrm{PR}+\mathrm{SD})$ caseload $/$ total caseload $\times 100 \%$, overall response rate $(\mathrm{ORR})=(\mathrm{CR}+\mathrm{PR})$ caseload $/$ total caseload $\times 100 \%{ }^{14}$

\section{Statistical methods}

Original case data were sorted using Microsoft Excel, and mean AUC, SD and median were calculated. Curve regression coefficient analysis was performed using Spearman. SPSS 19.0 software was used, and the $\chi^{2}$ test employed to compare 5-FU AUC, severe adverse reactions, and efficacy evaluation. $\mathrm{P}<0.05$ was considered statistically significant.

\section{RESULTS}

\section{General condition of patients}

This study included 47 cases with 28 men and 19 women (average age, $60 \pm 10$ years). The number of patients with colon, rectal-sigmoid junctional, and rectal cancer was 24, 5 and 23, respectively, and included five cases that successively received both chemotherapy regimens. The complicating diseases included diabetes, hypertension, sinus tachycardia, atrial fibrillation, lacunar infarction, and allergic dermatitis with caseloads of $12,14,2,2,1$ and 1 , respectively. There were 33 and 19 cases of first-line and second-line chemotherapy, respectively. The number of patients treated with mFOLFOX6, FOLFOX4 and FOLFIRI was 20, 7 and 15, respectively, and 32 cases were treated via combination with bevacizumab.

\section{DI, TI, RDI and AUC of 5-FU}

Chemotherapy was administered to the 47 cases and cumulatively, plasma concentration monitoring was performed 289 times; the average number of measurements per case was
5.9 \pm 3.1 times. Mean DI, TI and RDI reflecting 5-FU dose were $83.3 \% \pm 16.5 \%, 76.1 \% \pm 20.4 \%$ and $63.1 \% \pm 21.1 \%$, respectively. The AUC range was $2.8-158 \mathrm{mg}^{*} \mathrm{~h} / \mathrm{L}$, and mean AUC for each patient was $41 \pm 94.6 \mathrm{mg}$ *h/L, of which AUC of 60 cases $(20.8 \%)$ and average AUC of 13 patients $(27.7 \%)$ were within the target range (table 1). A Spearman linear regression analysis was performed on the average AUC of 5-FU for each patient and DI of 5-FU. The results suggested that 5-FU AUC increased with an increase in DI (figure 1).

\section{Correlation between AUC and RDI of 5-FU, and severe adverse reactions and curative effect of anti-tumour therapy}

Severe adverse reaction and effect of anti-tumour therapy for the AUC <20 mg*h/L, 20-30 mg*h/L and >30 mg*h/L patient groups are shown in table 2 . All differences in the incidence of 5-FU-related severe adverse reactions (neutropenia, anaemia, thrombocytopenia, diarrhoea, oral mucositis, handfoot syndrome), overall incidence of severe adverse reaction, DCR and ORR between the three groups were not statistically significant $(p>0.05)$. Overall incidence of severe adverse events was lower in the AUC $\leq 30 \mathrm{mg}$ " $h / L$ group (combination of the AUC $<20$ and $20-30 \mathrm{mg}^{*} \mathrm{~h} / \mathrm{L}$ groups; $24.0 \%$ ) than in the AUC $>30 \mathrm{mg}$ *h/L group (50.0\%); no statistical difference was found $(\mathrm{p}=0.06)$. The incidence of severe neutropenia was $12.0 \%$ and $40.9 \%$, respectively, and similarly, no statistical difference was found $(p=0.05)$. The DCR of the AUC $\geq 20 \mathrm{mg}^{*} \mathrm{~h} / \mathrm{L}$ group (combination of the AUC $20-30$ and $>30 \mathrm{mg} * \mathrm{~h} / \mathrm{L}$ groups; 97.1\%) was higher than that of the AUC $<20 \mathrm{mg}^{*} \mathrm{~h} / \mathrm{L}$ group $(83.3 \%)$; no statistical difference was found $(\mathrm{p}=0.09)$. The ORR of the two groups was $51.4 \%$ and $25.0 \%$, respectively, and no statistical difference was found $(\mathrm{p}=0.11)$.

The limit $68 \%$ is the median RDI value of overall level. Table 3 shows the severe adverse reaction and anti-tumour effect of 5 -FU RDI $<68 \%$ group and $\geq 68 \%$ group. DCR between the two groups was $86.4 \%$ and $100.0 \%$, respectively, with no statistical difference found $(\mathrm{p}=0.19)$. The 5 -FU RDI $<68 \%$ group had a lower ORR than the 5 -FU RDI $\geq 68 \%$ group $(31.8 \%$ and $56.0 \%$, respectively); we found no statistical difference $(p=0.10)$.

Table 3 The relationship between relative dose intensity and treatment outcomes

\begin{tabular}{|c|c|c|c|c|c|c|c|c|c|c|c|}
\hline \multirow[b]{2}{*}{$\begin{array}{l}\text { RDI } \\
\text { level }\end{array}$} & \multicolumn{7}{|c|}{ Curative effect case load (n) } & \multicolumn{4}{|c|}{ Curative effect case load (n) } \\
\hline & Neutropenia & Anaemia & Thrombocytopenia & Diarrhoea & $\begin{array}{l}\text { Oral } \\
\text { mucositis }\end{array}$ & $\begin{array}{l}\text { Hand-foot } \\
\text { syndrome }\end{array}$ & $\begin{array}{l}\text { Cases } \\
\text { having } \\
\text { severe } \\
\text { adverse } \\
\text { reactions } \\
\text { (n) }\end{array}$ & $\begin{array}{l}\text { Complete } \\
\text { response }\end{array}$ & $\begin{array}{l}\text { Partial } \\
\text { response }\end{array}$ & Stable & Progressive \\
\hline$<68$ & 8 & 2 & 3 & 3 & 3 & 0 & 12 & 0 & 7 & 12 & 3 \\
\hline$\geq 68$ & 4 & 0 & 0 & 0 & 4 & 0 & 5 & 2 & 12 & 11 & 0 \\
\hline
\end{tabular}

Curative effect case load $(\mathrm{n})$

$\mathrm{RDI}$, relative dose intensity. 
Table 4 Adjusting scheme of fluorouracil dosage according to the area under the receiver operating characteristic curve

\begin{tabular}{|c|c|c|c|c|c|c|c|c|c|}
\hline \multicolumn{2}{|c|}{ Gamelin (2008) } & \multicolumn{2}{|c|}{ Wasif (2009) } & \multicolumn{2}{|c|}{ Kaldate (2012) } & \multicolumn{2}{|c|}{ Wilhelm (2016) } & \multicolumn{2}{|c|}{ Denda (2016) } \\
\hline AUC & \pm Ratio (\%) & AUC & \pm Ratio (\%) & AUC & \pm Ratio (\%) & AUC & \pm Ratio (\%) & AUC & \pm Ratio(\%) \\
\hline$<4$ & 70 & $<5$ & 150 & $<8$ & 30 & $<8$ & Repeat the original dose, if the AUC is again $<8$, increase it by $30 \%$ & $<8$ & 30 \\
\hline $4-8$ & 50 & $5-10$ & 100 & $8-13$ & 24 & $8-13$ & 25 & $8-13$ & 25 \\
\hline $8-10$ & 40 & $10-15$ & 25 & $14-16$ & 18 & $14-16$ & 20 & $14-16$ & 20 \\
\hline $10-12$ & 30 & $15-20$ & 15 & $17-19$ & 12 & $17-19$ & 10 & $17-19$ & 10 \\
\hline $12-15$ & 20 & $20-25$ & Unchanged & $20-30$ & Unchanged & $20-29$ & Unchanged & $20-30$ & Unchanged \\
\hline 15-18 & 10 & $25-30$ & -10 & $30-33$ & -12 & $30-33$ & -10 & $30-33$ & -10 \\
\hline $18-20$ & 5 & $30-35$ & -15 & $34-36$ & -18 & $34-36$ & -20 & $34-36$ & -20 \\
\hline 20-24 & Unchanged & $35-40$ & -20 & $37-39$ & -24 & $37-39$ & -25 & $37-39$ & -25 \\
\hline $24-48$ & -5 & - & - & $\geq 40$ & -30 & $\geq 40$ & -30 & $\geq 40$ & -30 \\
\hline $28-31$ & -10 & - & - & - & - & - & - & - & - \\
\hline$>31$ & -15 & - & - & - & - & - & - & - & - \\
\hline
\end{tabular}

AUC, area under the receiver operating characteristic (ROC) curve.

\section{DISCUSSION}

\section{Technical points and clinical significance of 5-FU plasma concentration monitoring}

The techniques used to detect 5-FU TDM include highperformance liquid chromatography, liquid chromatography tandem mass spectrometry, and immunoassay, with each having their respective advantages and disadvantages. ${ }^{12}{ }^{15-18}$ In this study, 5-FU plasma concentration was determined via liquid chromatography tandem mass spectrometry that was strictly validated using a previous methodology. This technique is accurate and reliable for detection. ${ }^{12}$ The 5 -FU plasma concentration presents chronological characteristics, and fluctuation in plasma concentration appears during a single intravenous pumping of 5 -FU. ${ }^{19}$ In this study, the time of blood sample collection was uniformly controlled between 8:30 and 9:30 (4.5-5.5 hours before the end of intravenous pumping) to reduce the fluctuation in plasma concentration caused by the chronological characteristics of 5-FU. The premature end of venous pumping was avoided, and it was convenient for staff members to collect plasma samples during regular working hours.

Due to the poor stability of 5 -FU in whole blood, ${ }^{2021}$ it is necessary to centrifuge whole blood to extract plasma at the earliest convenience, or to place whole blood into a polyethylene tube with a stabiliser (5-FU metabolic enzyme dihydrouracil dehydrogenase inhibitor, gimeracil) after whole blood collection. Strict control was employed by the staff during plasma sample collection to prevent an overly-low result in the detection of 5-FU plasma concentration owing to its instability in whole blood. ${ }^{17} 18$

Gamelin et al proposed a programme to adjust 5-FU drug dose based on its AUC, as depicted in table 4. When AUC is lower than the target value, the subsequent 5-FU dose is increased, and when it is higher, the subsequent 5 -FU dose is decreased..$^{5-9}$ This group of patients possess a relatively broad target AUC range of 20-30 mg*h/L, which is consistent with relevant Chinese publications. ${ }^{15} 17$ AUC in multiple chemotherapy cycles was used to analyse the correlation between AUC and severe adverse reactions, and anti-tumour efficacy. We concluded that AUC levels were positively correlated with the incidence of severe adverse reactions and anti-tumour efficacy. The results obtained for this subject group are inherently consistent with the study by $\mathrm{Ju}$ et al. ${ }^{152}$ No statistical significance was found in this research group, which may be attributed to the overly-low number of cases included.

For most patients, 5-FU dose remained unchanged while AUC displayed a certain fluctuation range each time, suggesting that it is inappropriate to adjust 5-FU dose based on AUC level each time; this is consistent with the results of Matsumoto et al. ${ }^{19} 20$ Referring to the average value of 5-FU plasma concentration measured by Ju et al during multiple chemotherapy cycles, ${ }^{15} 22$ we combined 5-FU AUC with DI, TI, RDI and severe adverse reactions. The 5-FU dose adjustment scheme recommended by Denda in table 4 suggests the need for a comprehensive judgement.

We recommend: for patients in the AUC $<20 \mathrm{mg}$ * $\mathrm{h} / \mathrm{L}$ group, if a severe adverse reaction does not occur, the upper limit of 5-FU drug dose can be increased until RDI reaches $100 \%$, to improve anti-tumour efficacy; for patients in the AUC $>30 \mathrm{mg}$ " $\mathrm{h} / \mathrm{L}$ group, if a severe adverse reaction occurs, the subsequent 5 -FU dose can be reduced to improve medication safety; and for patients in the AUC $\leq 30 \mathrm{mg}$ " h/L group, if a severe adverse reaction occurs, oxaliplatin or irinotecan dose can be preferentially reduced, and 5 -FU dose may not be reduced temporarily to ensure 5 -FU antitumour efficacy, while improving medication safety.

\section{Role of DI, TI and RDI in 5-FU plasma concentration monitoring}

DI can visually reflect each medication intensity. Mean DI, TI and RDI of 5 -FU in this group were $83.3 \% \pm 16.5 \%, 76.1 \% \pm 20.4 \%$ and $63.1 \% \pm 21.1 \%$, respectively, suggesting that the underdosing of anti-tumour drugs should be addressed..$^{3-5}$ RDI is related to DI and TI numerically, and from a clinical view it is associated with the efficacy of anti-tumour therapy, and is related to drug resistance of cancer cells. Munker et al ${ }^{23}$ studied the effect of RDI on chemotherapy efficacy, and it was agreed that a relatively high RDI should be applied if adverse reactions are tolerable. ${ }^{3-5}$ In this study, the proportion of AUC $<20 \mathrm{mg}{ }^{*} \mathrm{~h} / \mathrm{L}$ was $40.5 \%$, which may be due to the relative low DI and RDI. The authors recommend that DI, TI and RDI be close to $100 \%$ when the patient's adverse reactions are tolerable. The ratio of AUC $\geq 20 \mathrm{mg}$ * $\mathrm{h} / \mathrm{L}$ may be raised and the efficacy of 5 -FU antitumour therapy can be further improved. When severe adverse reactions occur, 5-FU plasma concentration monitoring results should be considered, and chemotherapy dose may be adjusted to reduce the risk of severe adverse reactions and ensure antitumour efficacy. ${ }^{23}$

In conclusion, 5-FU plasma concentration monitoring may improve the safety and efficacy of treating patients with advanced colorectal cancer in China. It is expected to become important for clinical individualised 5-FU use. 
Funding This study was supported by Grant Scientific Research Projects of Changzhou Health and Family Planning Commission (No. ZD201514), Guiding Scientific Research Projects of Changzhou Health and Family Planning Commission (No. WZ201719), Project from Hubei Center for ADR Monitoring of Drugs and Medical Devices (No. 20160422) and funding for the research-oriented clinician plan of Tongji Medical College, Huazhong University of Science and Technology (No. 5001540076).

Competing interests None declared.

Patient consent for publication Not required.

Provenance and peer review Not commissioned; externally peer reviewed.

ORCID iD

Guangzhao He http://orcid.org/0000-0001-7706-9135

\section{REFERENCES}

1 Chen W, Zheng R, Baade PD, et al. Cancer statistics in China, 2015. CA Cancer J Clin 2016;66:115-32.

2 Elez $\mathrm{E}$, Argilés $\mathrm{G}$, Tabernero J. First-line treatment of metastatic colorectal cancer: interpreting FIRE-3, peak, and CALGB/SWOG 80405. Curr Treat Options Oncol 2015;16.

3 Nakayama G, Tanaka C, Uehara K, et al. The impact of dose/time modification in irinotecan- and oxaliplatin-based chemotherapies on outcomes in metastatic colorectal cancer. Cancer Chemother Pharmacol 2014;73:847-55.

4 Chen Y, LV J, YY X, et al. Review of rational use of antitumor drugs for colon cancer in our hospital. Central South Pharmacy 2017;15:1643-6.

5 Yang J, Yuan XH, Zhang XL, et al. Impact of dose intensity of first-line chemotherapy on prognosis of patients with metastatic colorectal cancer. Tumor 2017;37:255-61.

6 Gamelin E, Delva R, Jacob J, et al. Individual fluorouracil Dose Adjustment based on pharmacokinetic follow-up compared with conventional dosage: results of a multicenter randomized trial of patients with metastatic colorectal cancer. J Clin Oncol 2008;26:2099-105.

7 Saif MW, Choma A, Salamone SJ, et al. Pharmacokinetically guided Dose Adjustment of 5-fluorouracil: a rational approach to improving therapeutic outcomes. I Natl Cancer Inst 2009;101:1543-52.

8 Kaldate RR, Haregewoin A, Grier CE, et al. Modeling the 5-fluorouracil area under the curve versus dose relationship to develop a pharmacokinetic dosing algorithm for colorectal cancer patients receiving FOLFOX6. Oncologist 2012;17:296-302.

9 Wilhelm M, Mueller L, Miller MC, et al. Prospective, multicenter study of 5-fluorouraci therapeutic drug monitoring in metastatic colorectal cancer treated in routine clinical practice. Clin Colorectal Cancer 2016;15:381-8.
10 Denda T, Kanda M, Morita Y, et al. Pharmacokinetic Dose Adjustment of 5-FU in modified FOLFOX7 plus bevacizumab for metastatic colorectal cancer in Japanese patients: a-JUST phase II clinical trial. Cancer Chemother Pharmacol 2016;78:1253-61.

11 Beumer $\mathrm{JH}$, Chu E, Allegra C, et al. Therapeutic drug monitoring in oncology: international Association of therapeutic drug monitoring and clinical toxicology recommendations for 5-fluorouracil therapy. Clin Pharmacol Ther 2019;105.

$12 \mathrm{GZ} \mathrm{H}$, Xue HB, Yang QL, et al. Establishment of plasma concentration detection method for 5-fluorouracil and its clinical application. China Pharmacist 2017;20:49-52.

13 US Department of Health and Human Services. Common terminology criteria for adverse events (CTCAE) version 4.03, 2010. Available: https://evs.nci.nih.gov/ftp1/ CTCAE/CTCAE_4.03/CTCAE_4.03_20100614_QuickReference_5x7.pdf [Accessed 11 Feb 2019].

14 Eisenhauer EA, Therasse P, Bogaerts J, et al. New response evaluation criteria in solid tumours: revised RECIST guideline (version 1.1). Eur J Cancer 2009;45:228-47.

15 Ju Y, Zhou Y, Peng Y, et al. [Establishment and clinical application of liquid chromatography-tandem mass spectrometric method for simultaneous determination of plasma 5-fluorouracil]. Zhonghua Yi Xue Za Zhi 2016;96:817-21.

16 Moloney M, Faulkner D, Link E, et al. Feasibility of 5-fluorouracil pharmacokinetic monitoring using the My-5FU PCM ${ }^{\mathrm{TM}}$ system in a quaternary oncology centre. Cancer Chemother Pharmacol 2018;82:865-76.

17 Shi J, Chen HS, Duan CX, et al. Research status of therapeutic drug monitoring of 5-fluorouracil. Chin J Clin Pharmaco 2016;32:1053-6.

$18 \mathrm{GZ} \mathrm{H}$, Liu Q, XQ L, et al. Research progress of individualized fluoropyrimidines. Chin Hosp Pharm J 2018;6:679-83.

19 Matsumoto $\mathrm{H}$, Okumura $\mathrm{H}$, Murakami $\mathrm{H}$, et al. Fluctuation in plasma 5-fluorouracil concentration during continuous 5-fluorouracil infusion for colorectal cancer. Anticancer Res 2015;35:6193-9.

20 Lee JJ, Beumer JH, Chu E. Therapeutic drug monitoring of 5-fluorouracil. Cancer Chemother Pharmacol 2016;78:447-64.

21 Murphy RF, Balis FM, Poplack DG. Stability of 5-fluorouracil in whole blood and plasma. Clin Chem 1987;33:2299-300.

22 Cai $X$, Xue P, Song W-feng, et al. [Role of pharmacokinetic monitoring of serum fluorouracil concentration in patients with local advanced and metastatic colorectal cancer and further improving efficacy of fluorouracil-based chemotherapy]. Zhonghua Zhong Liu Za Zhi 2012;34:39-43.

23 Munker S, Gerken M, Fest P, et al. Chemotherapy for metastatic colon cancer: no effect on survival when the dose is reduced due to side effects. BMC Cancer 2018;18. 